\title{
Optimization and Structure-Activity
}

\section{Relationships of Phosphinic Pseudotripeptide}

\section{Inhibitors of Aminopeptidases that Generate}

\section{Antigenic Peptides.}

Paraskevi Kokkala, ${ }^{\dagger}$ Anastasia Mpakali, ${ }^{+}$Athanasios Papakyriakou, ${ }^{\ddagger}$ Ira Daskalaki, $^{\dagger}$ Ioanna Petropoulou, ${ }^{\dagger}$ Sofia Kavalou, ${ }^{\dagger}$ Mirto Papathanasopoulou, ${ }^{\dagger}$ Stefanos Agrotis, ${ }^{\dagger}$ Theodora-Markisia Fonsou, ${ }^{\dagger}$ Efstratios Stratikos ${ }^{*,+}$ and Dimitris Georgiadis ${ }^{*}{ }^{, \dagger}$

${ }^{\dagger}$ Department of Chemistry, Laboratory of Organic Chemistry, University of Athens, Panepistimiopolis, Zografou, 15771, Athens, Greece

'National Center for Scientific Research 'Demokritos', Aghia Paraskevi Attikis, GR 15310, Greece. 


\section{SUPPORTING INFORMATION}

\section{Table of contents}

1. Synthetic Methods and Characterizations S3

Aminoacid derivatives $\quad$ S3

Oxime derivatives $\quad 56$

2. RP-HPLC retention times and ES-MS data of inhibitors 6a-k, 13a-i, 14 and 22a-f S7

3. Titration of T-cell responses versus BMDCs exposed to different amounts of soluble ovalbumin

4. References 


\section{Synthetic Methods and Characterizations}

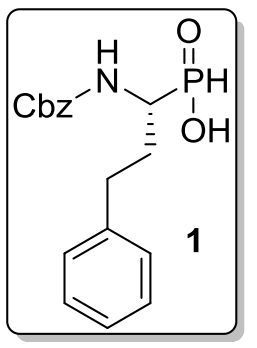

((1R)-1-\{[(Benzyloxy)carbonyl]amino\}-3-phenylpropyl)phosphinic acid (1). A racemic mixture of (1-\{[(benzyloxy)carbonyl]amino\}-3phenylpropyl)phosphinic acid (rac-1) (115 g, $345 \mathrm{mmol}$ ) prepared according to previously reported methods, ${ }^{1}$ was suspended in abs. EtOH $(980 \mathrm{~mL}$ ) and the mixture was refluxed. After complete dissolution, (S)-(-)$\alpha$-methylbenzylamine $(45 \mathrm{~mL}, 345 \mathrm{mmol}$ ) was added over a period of 10 min. After $15 \mathrm{~min}$, the mixture was slowly cooled at room temperature and then to $4{ }^{\circ} \mathrm{C}$. After 4 days at this temperature (with occasional stirring), the white precipitate was filtrated and the solid was washed with cold $\mathrm{EtOH}(3 \times 100 \mathrm{~mL})$ and $\mathrm{Et}_{2} \mathrm{O}(2 \times$ $100 \mathrm{~mL}$ ). The solid was dried over $\mathrm{P}_{2} \mathrm{O}_{5}$ and recrystallized by EtOH. After filtration, the solid was recrystallized once more to afford $33.8 \mathrm{~g}$ of the diastereoisomeric salt of 1 with (S)-(-)amine $\left([a]_{D}^{20}=-22.3, c=1, E t O H\right)$. The salt was stirred with $4 \mathrm{M} \mathrm{HCl}$ during $3 \mathrm{~h}$ and the pure phosphinic acid $1(24.7 \mathrm{~g}, 21 \%)\left([a]_{D}{ }^{20}=-35.7, \mathrm{c}=1, \mathrm{EtOH}\right)$ was isolated after filtration, washings with distilled water and drying in a desiccator over $\mathrm{P}_{2} \mathrm{O}_{5}$. A second batch of 1 (11.5 $\mathrm{g}, 10 \%)$ was obtained as follows: all filtrates from recrystallizations were concentrated, dried over $\mathrm{P}_{2} \mathrm{O}_{5}$, treated with $4 \mathrm{M} \mathrm{HCl}$ during $3 \mathrm{~h}$, filtrated and washed with distilled water. After drying over $\mathrm{P}_{2} \mathrm{O}_{5}$, the solid $(87 \mathrm{~g}, 261 \mathrm{mmol}$ ) was subjected to the same procedure as above, using $(R)-(+)-\alpha-$ methylbenzylamine. The filtrates obtained from the new round of recrystallizations were converted to the free acid (enriched to 1 ) which was treated with $(S)$ $(-)$ - $\alpha$-methylbenzylamine as described above. The obtained spectral data were found identical to literature data. ${ }^{1 \mathrm{~b}}$

\section{Aminoacid Derivatives:}

Aminoacid derivatives $\mathbf{5 a}, \mathbf{5} \mathbf{d}$ and $\mathbf{9 e}$ were commercially available. D-Phe derivative $\mathbf{5 k}$ was prepared according to the protocol employed for its enantiomer $\mathbf{5 c}$.

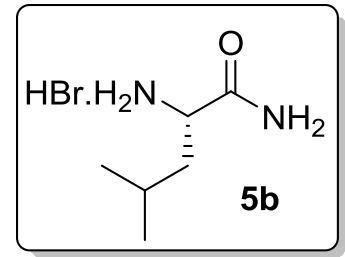

(S)-2-amino-4-methylpentanamide hydrobromide (5b). Cbz-(L)-Leu$\mathrm{OH}$ (760 mg, $2.86 \mathrm{mmol}$ ) was converted to Cbz-(L)-Leu-NH2 (501 mg, 66\%) according to the two-step procedure (methylation and substitution of resulting methylester by aqueous ammonia) reported by $\mathrm{Xu}$ et al.. ${ }^{2}$ A solution of Cbz-(L)-Leu-NH2 $(496 \mathrm{mg}, 1.88 \mathrm{mmol})$ in $33 \% \mathrm{HBr} / \mathrm{AcOH}(7.5 \mathrm{ml})$ was stirred at $\mathrm{rt}$ for $2 \mathrm{~h}$. After removal of the volatiles, compound $\mathbf{5 b}$ ( $334 \mathrm{mg}, 84 \%$ ) was obtained as a white solid after precipitation by EtOH. m.p. $238-240{ }^{\circ} \mathrm{C} ;[\alpha]_{D}{ }^{25}=+5.6^{\circ}\left(\mathrm{c}=0.5, \mathrm{H}_{2} \mathrm{O}\right) ;{ }^{1} \mathrm{H}$ NMR $\left(200 \mathrm{MHz}, \mathrm{D}_{2} \mathrm{O}\right) \delta 0.95(\mathrm{~d}, J=$ $4.6 \mathrm{~Hz}, 6 \mathrm{H}), 1.55-1.85(\mathrm{~m}, 3 \mathrm{H}), 4.04(\mathrm{t}, J=7 \mathrm{~Hz}, 1 \mathrm{H}) ;{ }^{13} \mathrm{C}$ NMR $\left(50 \mathrm{MHz}, \mathrm{D}_{2} \mathrm{O}\right) \delta$ 21.0, 22.0, 24.0, 39.9, 51.7, 172.9 .

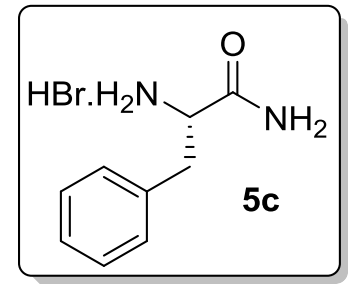

(S)-2- amino-3-phenylpropanamide hydrobromide (5c). Cbz-(L)-Phe$\mathrm{OH}(887 \mathrm{mg}, 2.96 \mathrm{mmol})$ was dissolved in AcOEt $(10 \mathrm{~mL})$ and 4nitrophenol (620 mg, $4.45 \mathrm{mmol})$, DIPEA (129 $\mu \mathrm{L}, 0.74 \mathrm{mmol})$ and EDC. $\mathrm{HCl}$ ( $843 \mathrm{mg}, 4.4 \mathrm{mmol}$ ) were added. After stirring at $\mathrm{rt}$ for $4 \mathrm{~h}$, AcOEt was evaporated in vacuo and the residue was diluted with $\mathrm{Et}_{2} \mathrm{O}(40 \mathrm{~mL})$. The resulting solution was washed with $2 \mathrm{M} \mathrm{HCl}(2 \times 10$ 
$\mathrm{mL}), 0.1 \mathrm{M} \mathrm{NaOH}(8 \times 10 \mathrm{~mL})$ and $\mathrm{H}_{2} \mathrm{O}(2 \times 10 \mathrm{~mL})$ and the organic phase was dried over $\mathrm{Na}_{2} \mathrm{SO}_{4}$ and evaporated in vacuo. The white solid obtained after precipitation by light petroleum (628 mg) was dissolved in THF ( $9 \mathrm{~mL}), 25 \%$ aq. $\mathrm{NH}_{3}(1.2 \mathrm{~mL}, 15 \mathrm{mmol})$ was slowly added at $0{ }^{\circ} \mathrm{C}$ and the resulting mixture was stirred at $\mathrm{rt}$ for $12 \mathrm{~h}$. After evaporation of the volatiles, the residue was diluted with $\mathrm{Et}_{2} \mathrm{O}(40 \mathrm{~mL})$ and the solution was washed with $1 \mathrm{M}$ $\mathrm{HCl}(2 \times 10 \mathrm{~mL}), \mathrm{H}_{2} \mathrm{O}(1 \times 10 \mathrm{~mL}), 0.1 \mathrm{M} \mathrm{NaOH}(9 \times 10 \mathrm{~mL})$ and $\mathrm{H}_{2} \mathrm{O}(2 \times 10 \mathrm{~mL})$. The organic phase was dried over $\mathrm{Na}_{2} \mathrm{SO}_{4}$ and evaporated in vacuo. Cbz-(L)-Phe-NH2 (400 mg, 45\%) was obtained as a white solid after trituration of the residue with $\mathrm{Et}_{2} \mathrm{O} / \mathrm{AcOEt}(1: 3) .{ }^{1} \mathrm{H}$ NMR (200 $\left.\mathrm{MHz}, \mathrm{DMSO}-d_{6}\right) \delta 2.75(\mathrm{dd}, J=10.6,13.6 \mathrm{~Hz}, 1 \mathrm{H}), 3.02(\mathrm{dd}, J=3.9,13.7 \mathrm{~Hz}, 1 \mathrm{H}), 4.19(\mathrm{dt}, J=$ 3.9, $10.6 \mathrm{~Hz}, 1 \mathrm{H}), 4.95(\mathrm{~s}, 2 \mathrm{H}), 7.09(\mathrm{br} \mathrm{s}, 1 \mathrm{H}), 7.15-7.38(\mathrm{~m}, 10 \mathrm{H}), 7.43(\mathrm{~d}, J=8.8 \mathrm{~Hz}, 1 \mathrm{H})$, 7.50 (br s, $1 \mathrm{H}) ;{ }^{13} \mathrm{C}$ NMR $\left(50 \mathrm{MHz}\right.$, DMSO-d $\left.d_{6}\right) \delta 37.6,56.1,65.2,126.3,127.5,127.7,128.1$, $128.3,129.2,137.1,138.3,155.9,173.5$. Cbz-(L)-Phe-NH2 (400 mg, $1.34 \mathrm{mmol}$ ) was deprotected as described above for the synthesis of $\mathbf{5 b}$. Compound $\mathbf{5 c}$ (301 $\mathrm{mg}, \mathbf{9 2 \%}$ ) was obtained as a white solid after precipitation by $\mathrm{Et}_{2} \mathrm{O}$. The obtained spectral data were found identical to literature data. ${ }^{3}$

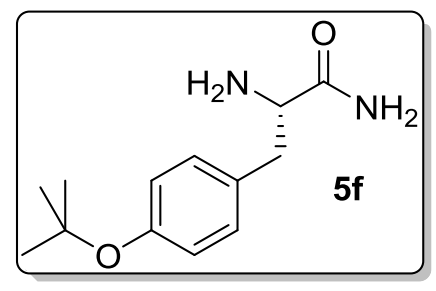

(S)-2-amino-3-[4-(tert-butoxy)phenyl]propanamide

$(5 f){ }^{4}$ Commercially available $\mathrm{HCl} \cdot \mathrm{H}-(\mathrm{L})-\mathrm{Tyr}\left(\mathrm{Bu}^{t}\right)$-OMe $(300 \mathrm{mg}, 1.04$ mmol) was suspended in water $(1.5 \mathrm{~mL}), 25 \%$ aq. $\mathrm{NH}_{3}(2.6 \mathrm{ml}$, $33 \mathrm{mmol}$ ) was added and the mixture was stirred for 72 hours. Extractions with DCM $(4 \times 10 \mathrm{~mL})$ followed and the organic phase was dried over $\mathrm{Na}_{2} \mathrm{SO}_{4}$ and evaporated in vacuo to afford amide $\mathbf{5 f}$ as a white solid (190 mg, 78\%). M.p. $121-124{ }^{\circ} \mathrm{C} ;{ }^{1} \mathrm{H}$ NMR (200 MHz, CD $\left.{ }_{3} \mathrm{OD}\right) \delta 1.29$ (s, 9H), 2.73 (dd, $1 \mathrm{H}, J=7.5,13.5 \mathrm{~Hz}$ ), 2.95 (dd, $1 \mathrm{H}, J=6.1,13.5 \mathrm{~Hz}$ ), 3.51 (dd, $1 \mathrm{H}, J=6.1,7.5$ $\mathrm{Hz}), 6.84-7.20(\mathrm{~m}, 4 \mathrm{H}) ;{ }^{13} \mathrm{C} \mathrm{NMR}(50 \mathrm{MHz}, \mathrm{CDCl} 3) \delta 28.9,40.3,56.6,78.5,124.4,129.7$, 132.6, 154.2, 177.9; ES-MS m/z: calcd for $\left[\mathrm{C}_{13} \mathrm{H}_{20} \mathrm{~N}_{2} \mathrm{O}_{2}+\mathrm{H}\right]^{+} 237.3$; found: 237.4 .

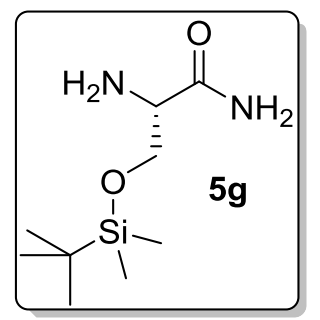

(S)-2-amino-3-[(tert-butyldimethylsilyl)oxy]propanamide $\quad(5 \mathrm{~g})$. The hydroxyl group of $\mathrm{Cbz}-(\mathrm{L})-\mathrm{Ser}-\mathrm{OH}(1.0 \mathrm{~g}, 4.2 \mathrm{mmol})$ was protected with the TBS protecting group according to the protocol of Lu et al.. ${ }^{5}$ The resulting TBS-derivative Cbz-(L)-Ser(TBS)-OH was converted to the amide $\mathrm{Cbz}-(\mathrm{L})-\mathrm{Ser}(\mathrm{TBS})-\mathrm{NH}_{2}$ by applying the two-step protocol used for the synthesis of $\mathbf{5 c}$ described above. $\mathrm{Cbz}-(\mathrm{L})-\mathrm{Ser}(\mathrm{TBS})-\mathrm{NH}_{2}$ was obtained as a white solid after trituration with $\mathrm{Et}_{2} \mathrm{O} / \mathrm{AcOEt}(1: 1)(585 \mathrm{mg}, 40 \%$ over three steps). M.p. $75-78^{\circ} \mathrm{C} ;[\alpha]_{\mathrm{D}}{ }^{25}=+35.1^{\circ}\left(\mathrm{c}=0.99, \mathrm{CHCl}_{3}\right) ;{ }^{1} \mathrm{H} \mathrm{NMR}\left(200 \mathrm{MHz}, \mathrm{CDCl}_{3}\right)$ $\delta 0.08 \& 0.09(2 \times \mathrm{s}, 6 \mathrm{H}), 0.89(\mathrm{~s}, 9 \mathrm{H}), 3.64(\mathrm{dd}, J=7.5,9.7 \mathrm{~Hz}, 1 \mathrm{H}), 4.06(\mathrm{dd}, J=3.8,9.7 \mathrm{~Hz}$, $1 \mathrm{H}), 4.14-4.28(\mathrm{~m}, 1 \mathrm{H}), 5.13(\mathrm{~s}, 2 \mathrm{H}), 5.57(\mathrm{br} \mathrm{s}, 1 \mathrm{H}), 5.70(\mathrm{~d}, J=4.9 \mathrm{~Hz}, 1 \mathrm{H}), 6.51(\mathrm{br} \mathrm{s}, 1 \mathrm{H})$, $7.30-7.40(\mathrm{~m}, 5 \mathrm{H}) ;{ }^{13} \mathrm{C}$ NMR $\left(50 \mathrm{MHz}, \mathrm{CD}_{3} \mathrm{OD}\right) \delta-5.3,19.0,26.3,57.7,64.5,67.7,79.3$, 128.8, 128.9, 129.3, 137.7, 157.9, 174.9; ES-MS m/z: calcd for $\left[\mathrm{C}_{17} \mathrm{H}_{28} \mathrm{~N}_{2} \mathrm{O}_{4} \mathrm{Si}+\mathrm{H}\right]^{+} 353.2$; found: 353.2 . A mixture of Cbz-(L)-Ser(TBS)- $\mathrm{NH}_{2}(400 \mathrm{mg}, 1.13 \mathrm{mmol}$ ) and $\mathrm{Pd} / \mathrm{C} 10 \%$ (40 mg) in $\mathrm{MeOH}\left(5 \mathrm{~mL}\right.$ ) was stirred at $\mathrm{rt}$ for $3 \mathrm{~h}$ in a $\mathrm{H}_{2}$ atmosphere. Then, the catalyst was removed by filtration through celite and the filtrates were concentrated to dryness affording $\mathbf{5 g}$ (205 $\mathrm{mg}, 83 \%)$ as a white solid. The obtained spectral data were found identical to literature data. $^{5}$ 


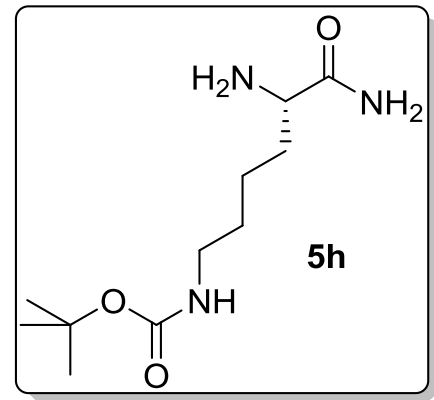

tert-Butyl (S)-(5,6-diamino-6-oxohexyl)carbamate (5h). Commercially available Cbz-(L)-Lys(Boc)-OH $(600 \mathrm{mg}, 1.58$ mmol) was converted to the amide $\mathrm{Cbz}-(\mathrm{L})-\mathrm{Lys}(\mathrm{Boc})-\mathrm{NH}_{2}$ by applying the two-step protocol used for the synthesis of $\mathbf{5 c}$ described above. Cbz-(L)-Lys(Boc)- $\mathrm{NH}_{2}$ (420 mg, 70\%) was obtained as a white solid and all spectral data were found identical to literature data. ${ }^{6}$ Hydrogenolysis of $\mathrm{Cbz}-(\mathrm{L})$ Lys(Boc)- $\mathrm{NH}_{2}$ (416 mg, $1.1 \mathrm{mmol}$ ) according to the protocol used for the preparation of $\mathbf{5 g}$ afforded $5 \mathrm{~h}(240 \mathrm{mg}, 89 \%)$ as a white solid. ${ }^{1} \mathrm{H}$ NMR $\left(200 \mathrm{MHz}, \mathrm{D}_{2} \mathrm{O}\right) \delta 1.07-1.91(\mathrm{~m}, 15 \mathrm{H}), 3.02(\mathrm{t}, J=6.2 \mathrm{~Hz}, 2 \mathrm{H}), 3.47-$ $3.75(\mathrm{~m}, 1 \mathrm{H}) ;{ }^{13} \mathrm{C} \mathrm{NMR}\left(50 \mathrm{MHz}, \mathrm{D}_{2} \mathrm{O}\right) \delta 21.8,27.8,28.8,32.5,39.7,53.6,80.9,158.4,177.0$.

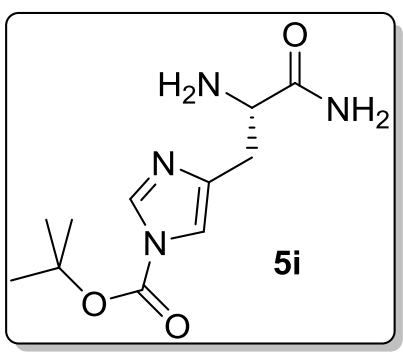

tert-Butyl (S)-4-(2,3-diamino-3-oxopropyl)-1H-imidazole-1carboxylate (5i). In a solution of commercially available $\mathrm{Cbz}-(\mathrm{L})$ His- $\mathrm{NH}_{2}$ (327 mg, $1.13 \mathrm{mmol}$ ) in DMF (4 mL), Boc ${ }_{2} \mathrm{O}$ (306 mg, 1.4 $\mathrm{mmol})$ in DMF $(0.4 \mathrm{~mL})$ was slowly added at $0^{\circ} \mathrm{C}$ and the resulting mixture was stirred at $\mathrm{rt}$ for $24 \mathrm{~h}$. Then, the mixture was diluted with $\mathrm{H}_{2} \mathrm{O}(20 \mathrm{~mL})$ and AcOEt $(30 \mathrm{~mL})$. The aqueous phase was separated and the organic phase was extracted with $0.5 \mathrm{M} \mathrm{HCl}(1 \times 10 \mathrm{~mL})$ and $\mathrm{H}_{2} \mathrm{O}(5 \times 10 \mathrm{~mL})$. The organic phase was dried over $\mathrm{Na}_{2} \mathrm{SO}_{4}$ and evaporated in vacuo to afford Cbz-(L)-His(Boc)- $\mathrm{NH}_{2}(205 \mathrm{mg}, 47 \%)$ as a white solid after precipitation by $\mathrm{Et}_{2} \mathrm{O} ;{ }^{7} \mathrm{~m} . \mathrm{p} 111-114{ }^{\circ} \mathrm{C} ;[\alpha]_{\mathrm{D}}{ }^{25}=+6.2^{\circ}(\mathrm{c}=0.42, \mathrm{MeOH}) ;{ }^{1} \mathrm{H}$ NMR $(200 \mathrm{MHz}$, $\left.\mathrm{CDCl}_{3}\right) \delta 1.54(\mathrm{~s}, 9 \mathrm{H}), 3.07(\mathrm{dd}, J=7.5,14.6 \mathrm{~Hz}, 1 \mathrm{H}), 3.32(\mathrm{dd}, J=3.3,14.6 \mathrm{~Hz}, 1 \mathrm{H}), 4.39-$ $4.61(\mathrm{~m}, 1 \mathrm{H}), 5.02(\mathrm{~s}, 2 \mathrm{H}), 5.65(\mathrm{br} \mathrm{s}, 1 \mathrm{H}), 6.49(\mathrm{~d}, J=7.3 \mathrm{~Hz}, 1 \mathrm{H}), 7.18-7.42(\mathrm{~m}, 6 \mathrm{H}), 7.56$ (br s, 1H), 8.58 (br s, $\left.1 \mathrm{H}) ;{ }^{13} \mathrm{C} \mathrm{NMR} \mathrm{(50} \mathrm{MHz,} \mathrm{CDCl}_{3}\right) \delta 27.9,30.3,54.4,67.0,86.3,115.2$, 128.1, 128.6, 136.3, 136.8, 138.3, 146.6, 156.4, 173.8; ES-MS m/z: calcd for $\left[\mathrm{C}_{19} \mathrm{H}_{24} \mathrm{~N}_{4} \mathrm{O}_{5}+\mathrm{H}\right]^{+}$ 389.2; found: 389.3. Hydrogenolysis of Cbz-(L)-His(Boc)- $\mathrm{NH}_{2}(200 \mathrm{mg}, 0.51 \mathrm{mmol}$ ) according to the protocol used for the preparation of $\mathbf{5 g}$ afforded $\mathbf{5 i}(115 \mathrm{mg}, 89 \%)$ as a white solid. ${ }^{1} \mathrm{H}$ $\operatorname{NMR}\left(200 \mathrm{MHz}, \mathrm{CDCl}_{3}\right) \delta 1.58(\mathrm{~s}, 9 \mathrm{H}), 2.51-3.35(\mathrm{~m}, 2 \mathrm{H}), 3.55-3.96(\mathrm{~m}, 1 \mathrm{H}), 5.97(\mathrm{br} \mathrm{s}, 1 \mathrm{H})$, 7.18 (s, 1H), 7.35 (br s, 1H), 8.00 (s, 1H); $\left.{ }^{13} \mathrm{C} \mathrm{NMR} \mathrm{(50} \mathrm{MHz,} \mathrm{CDCl}_{3}\right) \delta$ 27.9, 32.9, 55.0, 85.7, 114.8, 136.9, 140.0, 147.0, 177.5; ES-MS m/z: calcd for $\left[\mathrm{C}_{11} \mathrm{H}_{18} \mathrm{~N}_{4} \mathrm{O}_{3}+\mathrm{H}\right]^{+} 255.2$; found 255.3.

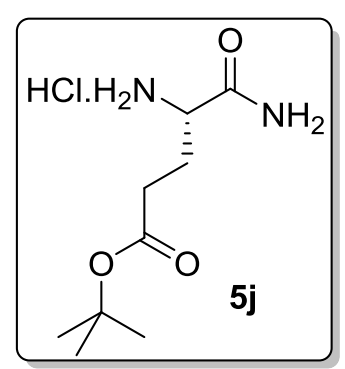

tert-Butyl (S)-(5,6-diamino-6-oxohexyl)carbamate (5j). Commercially available $\mathrm{Cbz}-(\mathrm{L})-\mathrm{Glu}\left(\mathrm{Bu}^{t}\right)-\mathrm{OH}(600 \mathrm{mg}, 1.78 \mathrm{mmol}$ ) was converted to the amide $\mathrm{Cbz}-(\mathrm{L})-\mathrm{Glu}\left(\mathrm{Bu}^{t}\right)-\mathrm{NH}_{2}$ by applying the two-step protocol used for the synthesis of $\mathbf{5 c}$ described above. $\mathrm{Cbz}-(\mathrm{L})-\mathrm{Glu}\left(\mathrm{Bu}^{t}\right)-\mathrm{NH}_{2}$ (419 mg, 70\%) was obtained as a white solid. M.p. $135-136{ }^{\circ} \mathrm{C}$; $[\alpha]_{\mathrm{D}}{ }^{25}=-5.3^{\circ}(\mathrm{c}=1, \mathrm{MeOH}) ;{ }^{1} \mathrm{H} \mathrm{NMR}\left(200 \mathrm{MHz}, \mathrm{CDCl}_{3}\right) \delta 1.43(\mathrm{~s}$, $9 \mathrm{H}), 1.83-2.20(\mathrm{~m}, 2 \mathrm{H}), 2.23-2.58(\mathrm{~m}, 2 \mathrm{H}), 4.24(\mathrm{dd}, J=7.8,12.6$ $\mathrm{Hz}, 1 \mathrm{H}), 5.55(\mathrm{br} \mathrm{s}, 1 \mathrm{H}), 5.73(\mathrm{~d}, J=7.3 \mathrm{~Hz}, 1 \mathrm{H}), 6.38(\mathrm{br} \mathrm{s}, 1 \mathrm{H}), 7.23-$ $7.43(\mathrm{~m}, 5 \mathrm{H}) ;{ }^{13} \mathrm{C}$ NMR $\left(50 \mathrm{MHz}, \mathrm{CDCl}_{3}\right) \delta 27.9,28.1,31.6,54.0,67.0,80.9,128.0,128.2$, 128.5, 136.2, 156.4, 172.7, 174.4; ES-MS m/z: calcd for $\left[\mathrm{C}_{17} \mathrm{H}_{24} \mathrm{~N}_{2} \mathrm{O}_{5}+\mathrm{H}\right]^{+} 337.2$; found: 337.2 . Hydrogenolysis of $\mathrm{Cbz}-(\mathrm{L})-\mathrm{Glu}\left(\mathrm{Bu}^{t}\right)-\mathrm{NH}_{2}(395 \mathrm{mg}, 1.17 \mathrm{mmol})$ according to the protocol used for the preparation of $5 \mathrm{~g}$ (with the addition of 5 drops of $2 \mathrm{M} \mathrm{HCl}$ to the reaction mixture) afforded $5 \mathbf{j}(249 \mathrm{mg}, 89 \%)$ as a white solid. $[\alpha]_{\mathrm{D}}{ }^{25}=+16.6^{\circ}\left(\mathrm{c}=0.5, \mathrm{H}_{2} \mathrm{O}\right) ;{ }^{1} \mathrm{H} \mathrm{NMR}(200 \mathrm{MHz}$, $\left.\mathrm{D}_{2} \mathrm{O}\right) \delta 1.42(\mathrm{~s}, 9 \mathrm{H}), 2.02(\mathrm{q}, J=7.0 \mathrm{~Hz}, 2 \mathrm{H}), 2.42(\mathrm{t}, J=7.2 \mathrm{~Hz}, 2 \mathrm{H}), 3.77(\mathrm{~m}, 1 \mathrm{H}) ;{ }^{13} \mathrm{C} \mathrm{NMR}(50$ 
$\left.\mathrm{MHz}, \mathrm{D}_{2} \mathrm{O}\right) \delta 27.3,31.0,52.8,83.0,174.1,174.7 ;$ ES-MS m/z: calcd for $\left[\mathrm{C}_{9} \mathrm{H}_{18} \mathrm{~N}_{2} \mathrm{O}_{3}+\mathrm{H}\right]^{+}$203.1; found: 203.2 .

\section{Oxime Derivatives:}

General procedure for conversion of aldehydes of type 10 to the corresponding oxime derivatives of type 11: A solution of an aldehyde of type $10(1 \mathrm{mmol})$, hydroxylamine hydrochloride (97 mg, $1.4 \mathrm{mmol}$ ) and sodium acetate $(164 \mathrm{mg}, 2 \mathrm{mmol})$ in $\mathrm{MeOH} / \mathrm{H}_{2} \mathrm{O}$ 3.5:1 was stirred at $\mathrm{rt}$ for $24 \mathrm{~h}$. After the reaction was completed, the volatiles were removed by evaporation. The residue was diluted with $\mathrm{Et}_{2} \mathrm{O}(30 \mathrm{~mL})$ and the organic phase was washed with $\mathrm{H}_{2} \mathrm{O}(3 \times 10 \mathrm{~mL})$, dried over anhydrous $\mathrm{Na}_{2} \mathrm{SO}_{4}$ and evaporated in vacuo. The target oximes of type 11 were obtained (yields $85-95 \%$ ) as mixtures of $E / Z$ isomers of sufficient purity to be used in the next step without further purification. Physical and spectroscopic data were in agreement with literature reported data, for compounds $1 \mathbf{1} a^{8}{ }^{8} \mathbf{1 1} \mathbf{b}-\mathbf{d},{ }^{8 a, 9}$ and 11e-g. ${ }^{10}$ For the synthesis of oximes $11 \mathbf{h}$ and $\mathbf{1 1 i}$, the above protocol was applied to TBSprotected aldehydes $\mathbf{1 0}$ and $\mathbf{1 0 i}$, respectively, prepared according to literature procedures. $^{11}$

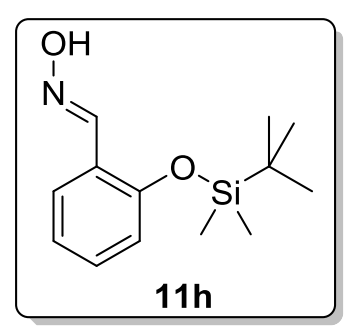

2-(tert-Butyldimethylsilyloxy)benzaldehyde oxime (11h). ${ }^{1} \mathrm{H}$ NMR $\left(200 \mathrm{MHz}, \mathrm{CDCl}_{3}\right) \delta 0.25(\mathrm{~s}, 6 \mathrm{H}), 1.03(\mathrm{~s}, 9 \mathrm{H}), 6.62-7.96(\mathrm{~m}, 5 \mathrm{H}), 8.51$ $(\mathrm{s}, 1 \mathrm{H}) ;{ }^{13} \mathrm{C}$ NMR $(50 \mathrm{MHz}, \mathrm{CDCl} 3) \delta-4.14,18.5,25.9,119.7,121.7$, 123.1, 126.6, 131.3, 146.8; ES-MS m/z: calcd for $\left[\mathrm{C}_{13} \mathrm{H}_{21} \mathrm{NO}_{2} \mathrm{Si}+\mathrm{H}\right]^{+}$ 252.1; found: 252.2 .

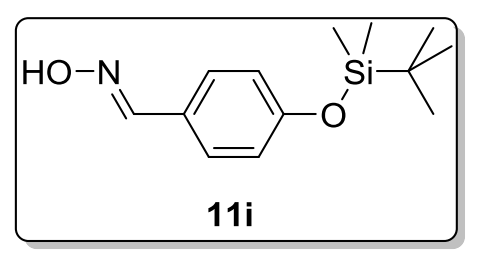

4-(tert-Butyldimethylsilyloxy)benzaldehyde oxime (11i). ${ }^{1} \mathrm{H}$ NMR $\left(200 \mathrm{MHz}, \mathrm{CDCl}_{3}\right) \delta 0.22(\mathrm{~s}, 6 \mathrm{H}), 0.99(\mathrm{~s}, 1 \mathrm{H}, 9 \mathrm{H}), 6.68-$ $7.13(\mathrm{~d}, 2 \mathrm{H}), 7.30-7.59$ (d, 2H), $8.11(\mathrm{~s}, 1 \mathrm{H}) ;{ }^{13} \mathrm{C}$ NMR (50 $\mathrm{MHz}, \mathrm{CDCl} 3) \delta-4.26,18.4,25.8,120.6,128.6,190.0$; ES-MS $\mathrm{m} / \mathrm{z}$ : calcd for $\left[\mathrm{C}_{13} \mathrm{H}_{21} \mathrm{NO}_{2} \mathrm{Si}+\mathrm{H}\right]^{+} 252.4$; found: 252.2 . 


\section{RP-HPLC retention times and ES-MS data of inhibitors $6 a-k, 13 a-i, 14$ and $22 a-f$}

\begin{tabular}{|c|c|c|c|c|c|}
\hline \multirow[t]{2}{*}{ Compound } & \multirow[t]{2}{*}{$t(\min )$} & \multirow[t]{2}{*}{ Gradient $^{1}$} & \multicolumn{3}{|c|}{ ES-MS $(m / z)^{2}$} \\
\hline & & & \multicolumn{2}{|l|}{ calcd for: } & found \\
\hline $6 a$ & 13.3 & $\mathrm{t}=0 \min (0 \% \mathrm{~B}), \mathrm{t}=25 \min (100 \% \mathrm{~B})$ & {$\left[\mathrm{C}_{19} \mathrm{H}_{32} \mathrm{~N}_{3} \mathrm{O}_{4} \mathrm{P}-\mathrm{H}\right]^{-}$} & 396.2 & 396.2 \\
\hline $6 b$ & 15.4 & $t=0 \min (0 \% B), t=25 \min (100 \% B)$ & {$\left[\mathrm{C}_{22} \mathrm{H}_{38} \mathrm{~N}_{3} \mathrm{O}_{4} \mathrm{P}-\mathrm{H}\right]^{-}$} & 438.3 & 438.3 \\
\hline $6 c$ & 16.8 & $t=0 \min (0 \% B), t=25 \min (100 \% B)$ & {$\left[\mathrm{C}_{25} \mathrm{H}_{36} \mathrm{~N}_{3} \mathrm{O}_{4} \mathrm{P}-\mathrm{H}\right]^{-}$} & 472.2 & 472.1 \\
\hline $6 \mathrm{~d}$ & 13.3 & $\mathrm{t}=0 \min (0 \% \mathrm{~B}), \mathrm{t}=25 \min (100 \% \mathrm{~B})$ & {$\left[\mathrm{C}_{21} \mathrm{H}_{34} \mathrm{~N}_{3} \mathrm{O}_{4} \mathrm{P}-\mathrm{H}\right]^{-}$} & 422.2 & 422.2 \\
\hline $6 e$ & 16.1 & $t=0 \min (0 \% B), t=25 \min (100 \% B)$ & {$\left[\mathrm{C}_{27} \mathrm{H}_{36} \mathrm{~N}_{4} \mathrm{O}_{5} \mathrm{P}-\mathrm{H}\right]^{-}$} & 511.3 & 511.4 \\
\hline $6 f$ & 13.8 & $t=0 \min (0 \% B), t=25 \min (100 \% B)$ & {$\left[\mathrm{C}_{25} \mathrm{H}_{36} \mathrm{~N}_{3} \mathrm{O}_{5} \mathrm{P}+\mathrm{H}\right]^{+}$} & 490.2 & 490.3 \\
\hline $6 g$ & 12.6 & $t=0 \min (0 \% B), t=25 \min (100 \% B)$ & {$\left[\mathrm{C}_{19} \mathrm{H}_{32} \mathrm{~N}_{3} \mathrm{O}_{5} \mathrm{P}-\mathrm{H}\right]^{-}$} & 412.2 & 412.3 \\
\hline $6 \mathrm{~h}$ & 12.1 & $t=0 \min (0 \% B), t=25 \min (100 \% B)$ & {$\left[\mathrm{C}_{22} \mathrm{H}_{39} \mathrm{~N}_{4} \mathrm{O}_{4} \mathrm{P}+\mathrm{H}\right]^{+}$} & 455.3 & 455.3 \\
\hline $6 i$ & 13.4 & $\mathrm{t}=0 \min (0 \% \mathrm{~B}), \mathrm{t}=25 \min (100 \% \mathrm{~B})$ & {$\left[\mathrm{C}_{22} \mathrm{H}_{34} \mathrm{~N}_{5} \mathrm{O}_{4} \mathrm{P}+\mathrm{H}\right]^{+}$} & 464.2 & 464.3 \\
\hline $6 \mathbf{j}$ & 12.6 & $t=0 \min (0 \% B), t=25 \min (100 \% B)$ & {$\left[\mathrm{C}_{21} \mathrm{H}_{34} \mathrm{~N}_{3} \mathrm{O}_{6} \mathrm{P}-\mathrm{H}\right]^{-}$} & 454.2 & 454.2 \\
\hline $6 k$ & 17.3 & $t=0 \min (0 \% B), t=25 \min (100 \% B)$ & {$\left[\mathrm{C}_{25} \mathrm{H}_{36} \mathrm{~N}_{3} \mathrm{O}_{4} \mathrm{P}-\mathrm{H}\right]^{-}$} & 472.2 & 472.1 \\
\hline $13 a^{3}$ & 11.3 & $\mathrm{t}=0 \min (30 \% \mathrm{~B}), \mathrm{t}=25 \min (100 \% \mathrm{~B})$ & {$\left[\mathrm{C}_{31} \mathrm{H}_{35} \mathrm{~N}_{4} \mathrm{O}_{5} \mathrm{P}-\mathrm{H}\right]^{-}$} & 573.2 & 573.3 \\
\hline 13b & 17.5 & $t=0 \min (15 \% B), t=25 \min (80 \% B)$ & {$\left[\mathrm{C}_{32} \mathrm{H}_{37} \mathrm{~N}_{4} \mathrm{O}_{6} \mathrm{P}-\mathrm{H}\right]^{-}$} & 603.3 & 603.4 \\
\hline $13 c$ & 17.2 & $\mathrm{t}=0 \min (15 \% \mathrm{~B}), \mathrm{t}=25 \min (80 \% \mathrm{~B})$ & {$\left[\mathrm{C}_{32} \mathrm{H}_{37} \mathrm{~N}_{4} \mathrm{O}_{6} \mathrm{P}-\mathrm{H}\right]^{-}$} & 603.3 & 603.3 \\
\hline 13d & 17.0 & $t=0 \min (15 \% B), t=25 \min (80 \% B)$ & {$\left[\mathrm{C}_{32} \mathrm{H}_{37} \mathrm{~N}_{4} \mathrm{O}_{6} \mathrm{P}-\mathrm{H}\right]^{-}$} & 603.3 & 603.3 \\
\hline $13 e$ & 18.3 & $t=0 \min (15 \% B), t=25 \min (80 \% B)$ & {$\left[\mathrm{C}_{31} \mathrm{H}_{34} \mathrm{ClN}_{4} \mathrm{O}_{5} \mathrm{P}-\mathrm{H}\right]^{-}$} & 607.2 & 607.3 \\
\hline $13 f$ & 19.1 & $t=0 \min (15 \% B), t=25 \min (80 \% B)$ & {$\left[\mathrm{C}_{31} \mathrm{H}_{34} \mathrm{ClN}_{4} \mathrm{O}_{5} \mathrm{P}-\mathrm{H}\right]^{-}$} & 607.2 & 607.3 \\
\hline $13 g$ & 19.0 & $t=0 \min (15 \% B), t=25 \min (80 \% B)$ & {$\left[\mathrm{C}_{31} \mathrm{H}_{34} \mathrm{ClN}_{4} \mathrm{O}_{5} \mathrm{P}-\mathrm{H}\right]^{-}$} & 607.2 & 607.4 \\
\hline $13 \mathrm{~h}$ & 17.1 & $t=0 \min (15 \% B), t=25 \min (80 \% B)$ & {$\left[\mathrm{C}_{31} \mathrm{H}_{35} \mathrm{~N}_{4} \mathrm{O}_{6} \mathrm{P}-\mathrm{H}\right]^{-}$} & 589.2 & 589.3 \\
\hline $13 \mathbf{i}$ & 14.0 & $t=0 \min (15 \% B), t=25 \min (80 \% B)$ & {$\left[\mathrm{C}_{31} \mathrm{H}_{35} \mathrm{~N}_{4} \mathrm{O}_{6} \mathrm{P}-\mathrm{H}\right]^{-}$} & 589.2 & 589.3 \\
\hline 14 & 14.6 & $t=0 \min (0 \% B), t=25 \min (100 \% B)$ & {$\left[\mathrm{C}_{24} \mathrm{H}_{30} \mathrm{~N}_{3} \mathrm{O}_{4} \mathrm{P}-\mathrm{H}\right]^{-}$} & 454.2 & 454.2 \\
\hline $22 a^{4}$ & $21.0 *$ & $\mathrm{t}=0 \min (30 \% \mathrm{~B}), \mathrm{t}=25 \min (70 \% \mathrm{~B})$ & {$\left[\mathrm{C}_{32} \mathrm{H}_{44} \mathrm{~N}_{3} \mathrm{O}_{4} \mathrm{P}+\mathrm{H}\right]^{+}$} & 566.3 & 566.4 \\
\hline $22 b^{5}$ & $18.5^{*}$ & $t=0 \min (30 \% B), t=25 \min (70 \% B)$ & {$\left[\mathrm{C}_{35} \mathrm{H}_{40} \mathrm{~N}_{3} \mathrm{O}_{4} \mathrm{P}+\mathrm{H}\right]^{+}$} & 598.3 & 598.3 \\
\hline $22 \mathrm{c}$ & $17.0^{* *}$ & $\mathrm{t}=0 \min (30 \% \mathrm{~B}), \mathrm{t}=25 \min (70 \% \mathrm{~B})$ & {$\left[\mathrm{C}_{32} \mathrm{H}_{42} \mathrm{~N}_{3} \mathrm{O}_{4} \mathrm{P}-\mathrm{H}\right]^{-}$} & 562.3 & 562.3 \\
\hline $22 d^{6}$ & $21.8^{*}$ & $\mathrm{t}=0 \min (40 \% \mathrm{~B}), \mathrm{t}=25 \min (70 \% \mathrm{~B})$ & {$\left[\mathrm{C}_{40} \mathrm{H}_{42} \mathrm{~N}_{3} \mathrm{O}_{4} \mathrm{P}+\mathrm{H}\right]^{+}$} & 660.3 & 660.2 \\
\hline $22 e$ & $15.4^{* *}$ & $t=0 \min (40 \% B), t=25 \min (100 \% B)$ & {$\left[\mathrm{C}_{37} \mathrm{H}_{44} \mathrm{~N}_{3} \mathrm{O}_{4} \mathrm{P}-\mathrm{H}\right]^{-}$} & 624.3 & 624.4 \\
\hline $22 f$ & $19.7^{* *}$ & $t=0 \min (40 \% B), t=25 \min (100 \% B)$ & {$\left[\mathrm{C}_{37} \mathrm{H}_{42} \mathrm{Cl}_{2} \mathrm{~N}_{3} \mathrm{O}_{4} \mathrm{P}-\mathrm{H}\right.$} & 692.2 & 692.2 \\
\hline
\end{tabular}

${ }^{1}$ Conditions: Hewlett-Packard 1100 model (C18-Cromasil-RP, $5 \mu \mathrm{m}$, UV/vis detector, flow:

$0.5 \mathrm{~mL} / \mathrm{min}, 254$ and/or $280 \mathrm{~nm}$ detection). Buffers used: A buffer: $90 \% \mathrm{H} 2 \mathrm{O}$ with $0.1 \%$ TFA, $10 \%$ CH3CN; B buffer: $10 \% \mathrm{H} 2 \mathrm{O}$ with $0.09 \%$ TFA, $90 \% \mathrm{CH} 3 \mathrm{CN}$.

${ }^{2}$ LC/MS analysis was performed to HPLC-purified isomers

${ }^{3}$ Retention time of its $\mathrm{P}_{1}{ }^{\prime}$-epimer, $\mathbf{1 3}^{\prime} \mathrm{a}^{\prime}$, by using the same method: $12.3 \mathrm{~min}$

${ }^{4}$ Retention time of its $\mathrm{P}_{1}{ }^{\prime}$-epimer, $\mathbf{2 2} \mathrm{a}^{\prime}$, by using the same method: $22.1 \mathrm{~min}$

${ }^{5}$ Retention time of its $\mathrm{P}_{1}{ }^{\prime}$-epimer, $\mathbf{2} \mathbf{2} \mathbf{b}^{\prime}$, by using the same method: $19.5 \mathrm{~min}$

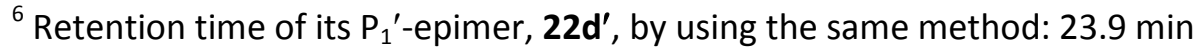


3. Titration of T-cell responses versus BMDCs exposed to different amounts of soluble ovalbumin

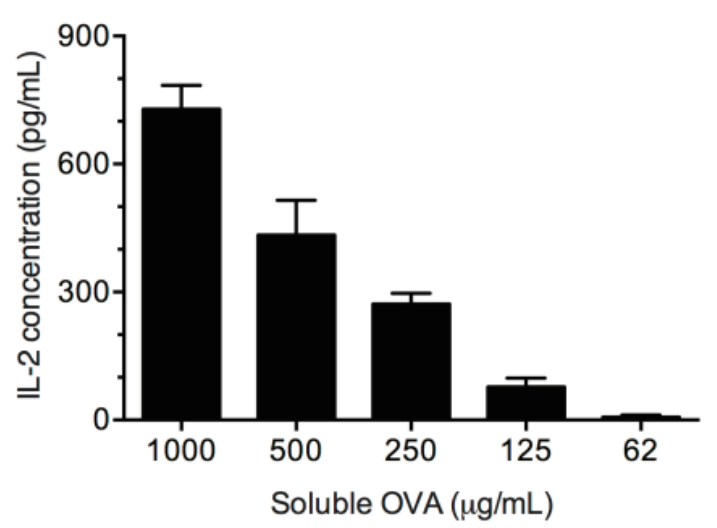

Figure S1: BMDCs isolated from wild-type mice were expose to different concentrations of soluble ovalbumin, incubated for 6 hours, fixed and then mixed with $\mathrm{CD}^{+}$T-cells isolated from the lymph nodes of Rag- $1^{-/-}$C57BI/6 OT-I mice. IL-2 secreted by the T-cells was measured by ELISA as described in the experimental methods section. 


\section{REFERENCES}

1. (a) Baylis, E. K.; Campbell, C. D.; Dingwall, J. G., 1-Aminoalkylphosphonous acids. Part 1. Isosteres of the protein amino acids. J. Chem. Soc., Perkin Trans. 1 1984, 2845-2853; (b) Grembecka, J.; Mucha, A.; Cierpicki, T.; Kafarski, P., The most potent organophosphorus inhibitors of leucine aminopeptidase. Structure-based design, chemistry, and activity. J. Med. Chem. 2003, 46 (13), 2641-2655.

2. Xu, Z.; DiCesare, J. C.; Baures, P. W., Parallel Synthesis of An Oligomeric Imidazole4,5-dicarboxamide Library. J. Comb. Chem. 2010, 12 (2), 248-254.

3. Moriguchi, T.; Yanagi, T.; Kunimori, M.; Wada, T.; Sekine, M., Synthesis and Properties of Aminoacylamido-AMP: Chemical Optimization for the Construction of an NAcyl Phosphoramidate Linkage. J. Org. Chem. 2000, 65 (24), 8229-8238.

4. Ager, D. J.; Prakash, I., Synthesis of aromatic amino acid amides. Synth. Commun. 1996, 26 (20), 3865-3868.

5. Lu, J.-Y.; Riedrich, M.; Wojtas, K.; Arndt, H.-D., Assembly of the Nosiheptide A-Ring: A Fruitful Lesson. Synthesis 2013, 45 (10), 1300-1311.

6. Routier, S.; Cotelle, N.; Catteau, J.-P.; Bernier, J.-L.; Waring, M. J.; Riou, J.-F.; Bailly, C., Salen-anthraquinone Conjugates. Synthesis, DNA-binding and cleaving properties, effects on topoisomerases and cytotoxicity. Bioorg. Med. Chem. 1996, 4 (8), 1185-1196.

7. Bergeron, R. J.; Ludin, C.; Müller, R.; Smith, R. E.; Phanstiel Iv, O., Development of a Hypusine Reagent for Peptide Synthesis. J. Org. Chem. 1997, 62 (10), 3271-3284.

8. (a) Hou, Y.; Lu, S.; Liu, G., lodine(III)-Mediated [3 + 2] cyclization for one-pot synthesis of benzo[d]isoxazole-4,7-diols in aqueous medium. J. Org. Chem. 2013, 78 (17), 8386-8395; (b) Puerto Galvis, C. E.; Kouznetsov, V. V., An unexpected formation of the novel 7-oxa-2-azabicyclo[2.2.1] hept-5-ene skeleton during the reaction of furfurylamine with maleimides and their bioprospection using a zebrafish embryo model. Org. Biomol. Chem. 2013, 11 (3), 407-411.

9. Patil, V. V.; Gayakwad, E. M.; Shankarling, G. S., Highly efficient and stable peracid for rapid and selective oxidation of aliphatic amines to oximes. New J. Chem. 2015, 39 (8), 6677-6682.

10. Zhang, L.; Chen, H.; Zha, Z.; Wang, Z., Electrochemical tandem synthesis of oximes from alcohols using $\mathrm{KNO}_{3}$ as the nitrogen source, mediated by tin microspheres in aqueous medium. Chem. Commun. 2012, 48 (52), 6574-6576.

11. Schmidt, B.; Hölter, F.; Kelling, A.; Schilde, U., Pd-catalyzed arylation reactions with phenol diazonium salts: Application in the synthesis of diarylheptanoids. J. Org. Chem. 2011, 76 (9), 3357-3365. 